\title{
NARROW-ANGLE AND WIDE-ANGLE ASTROMETRY VIA LONG BASELINE OPTICAL/INFRARED INTERFEROMETERS
}

\author{
XIAOPEI PAN, SHRI KULKARNI \\ California Institute of Technology, Pasadena, CA 91125 \\ AND \\ MICHAEL SHAO, M. MARK COLAVITA \\ Jet Propulsion Laboratory, Pasadena, CA 91109
}

\begin{abstract}
Long baseline optical/infrared interferometers, such as the Mark III Stellar Interferometer ${ }^{1}$ on Mt. Wilson and the ASEPS-0 Testbed Interferometer ${ }^{2}$ on Palomar Mountain, California, have good capabilities for narrow-angle and wide-angle astrometry with very high precision. Using the Mark III Interferometer many spectroscopic binaries became "visual" for the first time. The measurement accuracy of angular separation is 0.2 mas, the smallest separation measured between two components is 2 mas, the maximum magnitude difference is $4 \mathrm{mag}$, and the smallest semimajor axis is 4 mas. Such high angular resolution and dynamic range have been used to determine stellar masses with precision of $2 \%$ and differential stellar luminosities to better than $0.05 \mathrm{mag}$ for separations of less than $0 .{ }^{\prime \prime} 2$. For some binary stars, not only have the systems been resolved, but also the diameter of the primary component has been determined, yielding direct measurements of stellar effective temperature with high accuracy. For parallax determination, the precision is 1 mas or better and is unaffected by interstellar extinction. For wide-angle astrometry with the Mark III interferometer, the observation results yielded average formal $1 \sigma$ errors for FK5 stars of about 10 mas. Presently a new infrared interferometer, the ASEPS 0 Testbed Interferometer on Palomar Mountain is under construction, and is being optimized to perform high accuracy narrow-angle astrometry using long baseline observations at $2.2 \mu \mathrm{m}$, with phase referencing for increased sensitivity. The goal is to demonstrate differential astrometric accuracies of
\end{abstract}


0.06-0.1 $\mathrm{mas}^{3}$ in order to allow for detection of extra-solar planets in the near future.

Key words: astrometry - optical/IR interferometer

\section{Introduction}

Long baseline optical interferometers are the only instruments which are capable of both narrow-angle and wide-angle astrometry with milliarcsec precision. The Mark III Stellar Interferometer is a good example of such an instrument with important features which include active fringe tracking, two color astrometry, and full automation. This instrument has two fixed baselines (12 $\mathrm{m} \mathrm{N}$-S and E-S) for wide-angle astrometry, and a variable baseline $(3-32 \mathrm{~m} \mathrm{~N}-\mathrm{S})$ for narrow-angle astrometry. Typical measurements of fringe visibility are simultaneously made at $800 \mathrm{~nm}, 550 \mathrm{~nm}$, and $500 \mathrm{~nm}$ with a bandwidth of $22 \mathrm{~nm}, 25 \mathrm{~nm}$ and $25 \mathrm{~nm}$ respectively. The Mark III Interferometer measures $160-220$ stars per night and was in daily operation for more than 7 years. Brief descriptions of results for narrow- and wideangle astrometry will be given in the following sections.

\section{Parallax with Precision of $<5 \%$}

Parallax is one of the fundamental parameters for a star. Trigonometric parallax achieves a typical precision of 10 mas with the classical photographic technique. New CCD parallax programs have reached a precision at the 1 mas level. However it is limited to very faint stars (15 mag or dimmer) because it is extremely difficult to find reference stars in the small field of view. Another technique, the Multichannel Astrometric Photometer, provides relative parallax to the 1 mas level for many interesting bright stars. However, it is a difficult task to convert the relative parallax to the absolute value. For binary stars, the Mark III has determined the angular semi-major axis to 0.2 mas $^{4,5,6}$, and has yielded parallaxes with a precision of 1 mas after incorporating the linear semi-major axis from spectroscopy. This absolute parallax is not affected by interstellar absorption, and can be used as a calibrator and a check with HIPPARCOS's results. The largest distance obtained is $270 \mathrm{pc}$, corresponding to a parallax of 3.7 mas. Some results of parallax determination are listed in Table 1, and are compared with the corresponding values from the photographic techniques. It is interesting to note that the brightest binary in the Hyades cluster, $\theta^{2}$ Tau, has its distance determined as $44.1 \mathrm{pc} \pm 2.2 \mathrm{pc}$. This star is about $1 \mathrm{pc}$ in front of the center of the cluster. The estimated distance of the center of the Hyades is $45.5 \mathrm{pc}$, which is consistent with the latest results from other techniques. 
Table 1. Parallax Determination using the Mark III Interferometer

\begin{tabular}{lrrr} 
& \multicolumn{2}{c}{ Semimajor } & \multicolumn{2}{c}{ Parallax (mas) } \\
Star & \multicolumn{1}{c}{ Axis $a^{\prime \prime}$ (mas) } & \multicolumn{1}{c}{ Mark III } & Phgr. \\
HR 15 & $24.15 \pm 0.13$ & $29.6 \pm 1.0$ & 24 \\
HR 154 & $6.82 \pm 0.16$ & $3.7 \pm 0.1$ & 0 \\
HR 271 & $10.81 \pm 0.20$ & $14.3 \pm 0.6$ & 9 \\
HR 553 & $37.02 \pm 0.23$ & $58.1 \pm 1.1$ & 63 \\
HR 622 & $9.02 \pm 0.19$ & $30.6 \pm 1.3$ & 12 \\
HR 936 & $94.61 \pm 0.22$ & $35.4 \pm 1.1$ & 31 \\
HR 1412 & $18.60 \pm 0.18$ & $22.4 \pm 1.1$ & 29 \\
HR 5054 & $10.74 \pm 0.13$ & $44.1 \pm 0.8$ & 37 \\
HR 5793 & $7.75 \pm 0.13$ & $38.9 \pm 0.9$ & 43 \\
HR 6927 & $122.5 \pm 0.20$ & $118.7 \pm 1.0$ & 120 \\
HR 7478 & $23.7 \pm 0.40$ & $12.9 \pm 0.2$ & 7 \\
HR 8131 & $11.99 \pm 0.08$ & $18.1 \pm 0.8$ & 13
\end{tabular}

\section{Stellar Masses with $2 \%$ Accuracy}

The mass of a star determines its fate and evolutionary course. Stellar masses with an accuracy of 1-2\% are very critical to check evolutionary theory and modeling. There are fewer than 150 individual stars in binary systems with masses determined to better than $15 \%$, and only 45 stars have masses with a precision of $2 \%$. However, most of these stars are in the main sequence, and the evolved stars are very rare. The Mark III has determined inclinations ${ }^{7,8}$ to better than $1 \%$, and provided stellar masses to about $2 \%$; most of these stars are giants and sub-giants which are lacking among current measurements.

Table 2. Accurate Stellar Masses with the Mark III Interferometer

\begin{tabular}{cccccccc} 
Star & Spectrum & \multicolumn{1}{c}{$a^{\prime \prime}$ (mas) } & $i^{\circ}$ & $K_{1}(\mathrm{~km} / \mathrm{s})$ & $K_{2}(\mathrm{~km} / \mathrm{s})$ & $m_{1}\left(m_{\odot}\right)$ & $m_{2}\left(m_{\odot}\right)$ \\
HR5793 & B9.5IV,G5 & $\mathbf{7 . 7 5} \pm 0.13$ & $\mathbf{8 8 . 3 0} \pm 0.07$ & $35.35 \pm 0.50$ & $99.00 \pm 0.50$ & $2.58 \pm 0.04$ & $0.92 \pm 0.0$ \\
HR6927 & F7V,K3V & $122.5 \pm 0.20$ & $\mathbf{7 4 . 7 6} \pm 0.05$ & $17.87 \pm 0.10$ & $24.20 \pm 0.20$ & $1.08 \pm 0.03$ & $0.78 \pm 0.0$ \\
HR7478 & G8III,G8III & $\mathbf{2 3 . 7} \pm 0.40$ & $78.37 \pm 0.40$ & $26.79 \pm 0.05$ & $27.88 \pm 0.05$ & $2.26 \pm 0.05$ & $2.17 \pm 0.0$
\end{tabular}

\section{Precise Photometry within a Separation of $0 .{ }^{\prime \prime} 1$}

It has often been noted in the literature that there is a lack of precise measurements of stellar luminosities and colors along with mass determination. Interferometer is the only technique, besides Lunar Occultation, which can provide direct photometric measurements within small angular separations. In the periodic changes of fringe visibility of a binary star as measured with an interferometer, the peak-to-peak change is proportional 
to the intensity ratio of the two components. Since the typical precision of visibilities can reach $2 \%$ with the Mark III, the corresponding magnitude differences are readily determined to a precision of better than 0.05 mag. Another important characteristics of the Mark III is simultaneous measurements at multiple wavelengths. It is easy to calculate the color index for both components when combined with classical photometric results. It is worth noting that the traditional spectrophotometric method of analyzing spectral lines has had obvious biases as large as 1 mag. In contrast, lunar occultations do provide accurate magnitude differences for some binary systems, which agree well with the results of the Mark III Interferometer ${ }^{7}$. The comparisons between the Mark III and spectrophotometry are provided in Table 3.

Table 3. Comparison of Magnitude Differences measured with the Mark III Interferometer and Spectrophotometry

\begin{tabular}{|c|c|c|c|}
\hline \multirow[t]{2}{*}{ Star } & \multicolumn{2}{|c|}{ Mark III } & Spectrophotometry \\
\hline & at $800 \mathrm{~nm}$ & at $550 \mathrm{~nm}$ & at $550 \mathrm{~nm}$ \\
\hline HR 15 & $1 .^{m} 82 \pm 0 .^{m} 04$ & $1 .^{m} 99 \pm 0 .^{m} 04$ & $1 .^{m} 35$ \\
\hline HR 154 & $0 .^{m} 97 \pm 0 .^{m} 10$ & $0 .^{m} 92 \pm 0 .^{m} 10$ & $3 .^{m} 17$ \\
\hline HR 271 & $0 \cdot^{m} 94 \pm 0 .^{m} 06$ & $1 .^{m} 01 \pm 0 .^{m} 05$ & $0 .^{m} 29$ \\
\hline HR 553 & $2 .^{m} 63 \pm 0 . \cdot^{m} 22$ & $3 .^{m} 30 \pm 0 .^{m} 30$ & $2 .^{m} 80$ \\
\hline HR 622 & $0 .^{m} 52 \pm 0 .^{m} 04$ & $0 .^{m} 44 \pm 0 .^{m} 04$ & $1 .^{m} 19$ \\
\hline HR 936 & $2 .^{m} 63 \pm 0 \cdot^{m} 09$ & $2 .^{m} 92 \pm 0 .^{m} 15$ & $2 .^{m} 60$ \\
\hline HR 6927 & $2 .^{m} 02 \pm 0 .^{m} 06$ & $2 .^{m} 44 \pm 0 .^{m} 17$ & $1 .^{m} 99$ \\
\hline HR 8131 & $1 .^{m} 23 \pm 0 .^{m} 03$ & $0 .^{m} 47 \pm 0 .^{m} 06$ & $0 .^{m} 60$ \\
\hline
\end{tabular}

\section{Resolution of Radio Stars}

Radio stars play important roles in connecting stars with the extragalactic reference frame. VLBI observations have constructed a quasi-inertial reference frame to an accuracy of about $0 .{ }^{\prime \prime} 001$. The only way to link the optical reference frame to the radio reference frame is to observe these radio stars at both optical and radio wavelengths. The HIPPARCOS mission emphasized necessity to observe radio stars. However, many radio stars are binaries or in a triple system, and their orbital motion is not negligible if astrometric precision at the milliarcsec level is necessary.

The Mark III Interferometer has successfully resolved two radio stars ${ }^{9}$, Algol and $\lambda$ And. For the well-known triple system Algol, the AB-C system has had its geometric and physical parameters determined with higher resolution and accuracy than obtained with other techniques. Since the presence of the third component seriously complicates the analysis of both the light 
curves and the spectra of the central eclipsing pair, the results from the Mark III are very useful. The mass ratio $(r=2.63 \pm 0.20)$ indicates that the central pair will have displacements as large as 52 mas, which must be accounted for. For $\lambda$ And, the angular separations are about 3 mas, and the magnitude difference is about $4.5 \mathrm{mag}$ at $550 \mathrm{~nm}$.

\section{Wide-Angle Astrometry}

Modern meridian circles are the main astrometric instruments for measuring star positions at present, and have reached precisions of $0 .{ }^{\prime \prime} 1$ to $0 .{ }^{\prime \prime} 2$ for a single star transit. The analysis of observations from meridian circles has disclosed systematic errors in R.A. and Decl. in the Fundamental Star Catalogue (FK5) which reach about 0."1. Astrometric observations with the Mark III interferometer in $1988^{10}$ demonstrated average formal $1 \sigma$ errors of 6 mas in Decl. and $0.6 \mathrm{~ms}(=9 \mathrm{mas})$ in R.A. for a group of stars covering a range of $90^{\circ}$ in R.A. and $45^{\circ}$ in Decl. Such high precision comes from the good thermal control of the instrument, monitoring of variations of the delay offset, and the two color data analysis technique. Repeated observation were made in 1989 and had the same average formal $1 \sigma$ errors as in 1988. Examination of the errors for the difference of the positions for the two years (see Table 4 and the figures below) indicates that the formal errors of each year are underestimated by a factor of 1.5 , suggesting average external errors of approximately 9 mas in Decl. and 13 mas in R.A. for each year. As expected, observations with the Mark III Interferometer indicate that most of the FK5 stars in this group have positions within their estimated error ranges (about 50 mas in both coordinates in 1988 and 1989), and some stars do have position errors as large as 100 mas at present.

Table 4. Wide-angle Measurements of FK5 Stars in 1988 \& 1989 with the Mark III Interferometer

\begin{tabular}{rrrrrcrrr} 
FK5 & \multicolumn{3}{c}{ Offset in R.A.(ms) } & \multicolumn{3}{c}{ Offset in Decl.(mas) } \\
& 1988 & 1989 & Mean & \multicolumn{1}{c}{$\Delta$} & 1988 & 1989 & Mean & $\Delta$ \\
19 & $-2.0 \pm 0.5$ & $-0.7 \pm 0.5$ & -1.3 & -1.3 & $-2 \pm 5$ & $-18 \pm 6$ & -10 & 16 \\
33 & $-1.8 \pm 0.6$ & $-1.7 \pm 0.6$ & -1.7 & -0.1 & $15 \pm 5$ & $13 \pm 6$ & 14 & 2 \\
52 & $-2.2 \pm 0.7$ & $-2.7 \pm 0.8$ & -2.5 & 0.5 & $25 \pm 6$ & $29 \pm 8$ & 27 & -4 \\
66 & $7.0 \pm 0.5$ & $6.7 \pm 0.6$ & 6.8 & 0.3 & $3 \pm 6$ & $14 \pm 8$ & 9 & -11 \\
79 & $-0.5 \pm 0.6$ & $1.7 \pm 1.0$ & 0.6 & -2.2 & $-90 \pm 6$ & $-124 \pm 10$ & -107 & 34 \\
835 & $2.4 \pm 0.5$ & $2.1 \pm 0.4$ & 2.3 & 0.3 & $66 \pm 5$ & $80 \pm 4$ & 73 & -14 \\
848 & $6.2 \pm 0.7$ & $4.3 \pm 0.6$ & 5.2 & 1.9 & $-46 \pm 6$ & $-43 \pm 5$ & -45 & -3 \\
862 & $-5.3 \pm 0.5$ & $-4.2 \pm 0.4$ & -4.7 & -1.1 & $-35 \pm 5$ & $-32 \pm 4$ & -34 & -3 \\
1534 & $2.5 \pm 0.6$ & $0.3 \pm 0.3$ & 1.4 & 2.2 & $-10 \pm 5$ & $-7 \pm 4$ & -9 & -3 \\
1568 & $0.6 \pm 0.7$ & $-0.7 \pm 0.5$ & -0.0 & 1.3 & $25 \pm 5$ & $34 \pm 4$ & 30 & -9 \\
1619 & $-5.8 \pm 0.7$ & $-6.4 \pm 0.7$ & -6.1 & 0.6 & $-7 \pm 5$ & $2 \pm 7$ & -3 & -9
\end{tabular}



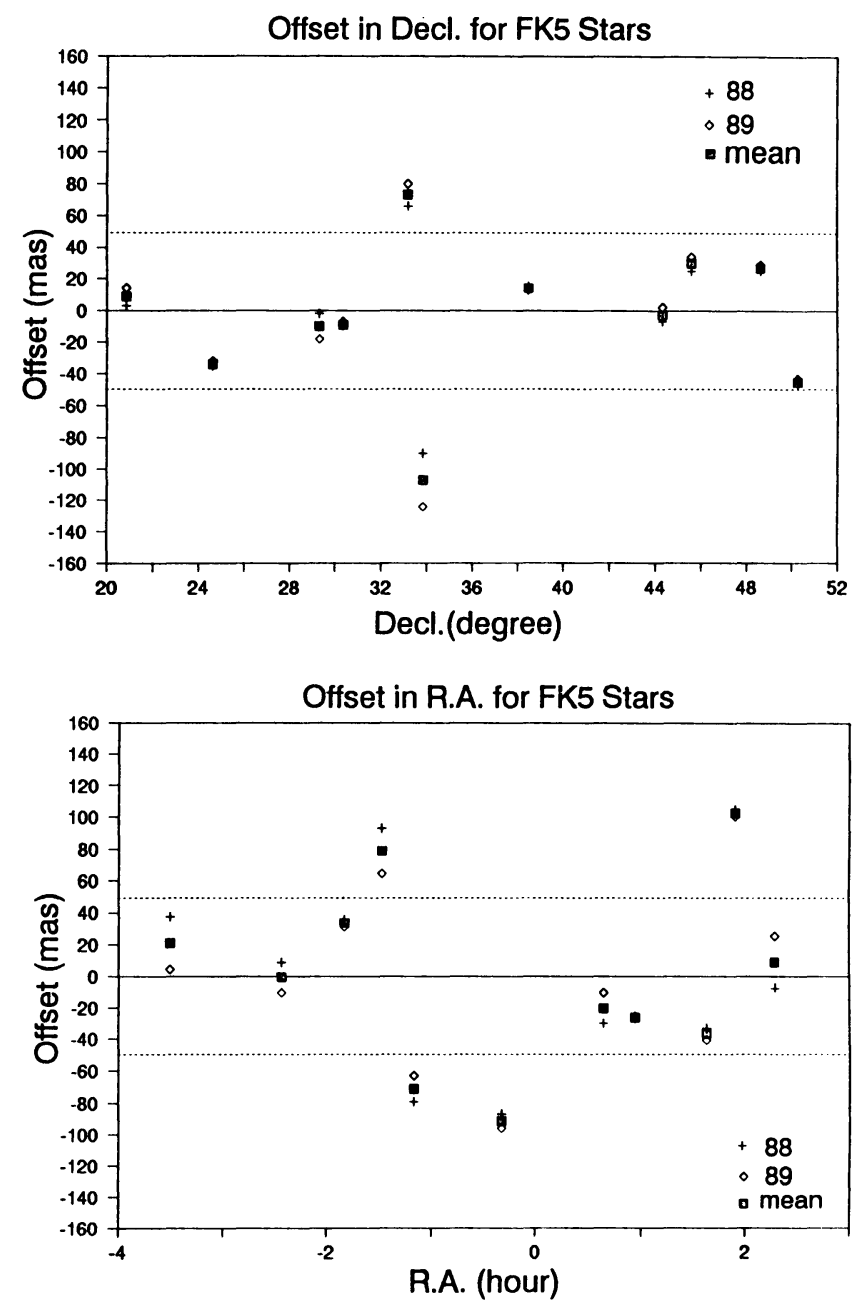

\section{References}

1. Shao, M. et al. 1988, A \& A, 193, 357

2. Colavita, M. M. et al. 1994, Proc. SPIE, 2200, 89

3. Colavita, M. M. 1994, A \& A, 283, 1027

4. Pan, X.P. et al. 1992, ApJ, 384, 624

5. Pan, X.P. et al. 1990, Proc. SPIE, 1237, 301

6. Pan, X.P. et al. 1993, IAU Coll. 135, 502

7. Pan, X.P. et al. 1994, Pro. SPIE, 2200, 360

8. Armstrong, J.T. et al. 1992, AJ, 104, 2217

9. Pan, X.P. et al. 1993, ApJ, 413, L129

10. Shao, M. et al. 1990, AJ, 100, 1701 